\title{
Modern Natural Sciences Could Inspire from Classical Chinese Metaphysics to Better Understand the Nature of Being
}

\author{
Maria Ferancova (Marina Čarnogurská)
}

Slovak Philosopher and Sinologue in Retirement, Slovak Republic

Copyright $\bigcirc 2016$ by authors, all rights reserved. Authors agree that this article remains permanently open access under the terms of the Creative Commons Attribution License 4.0 International License

\begin{abstract}
According to the latest scientific estimates, all resources needed for survival of Humankind on Earth are already in a deficit of up to one and a half planet! To slow down this rapidly widening deficit we are morally obliged to correct our behaviors not only from one to another but, most of all, toward the Earth! For that reason a radical reevaluation of many misinterpretations in present astrophysics, quantum theory and common natural sciences (also because of some incorrect religious opinions) are required, too. To search for a fundamental model to solve these problems is therefore necessary not only to find the most modern scientific, metaphysical and astrophysical solutions but also with the help of a comparative analysis to consider answers to similar questions by some ancient cultures and civilizations on Earth. Hoping to be inspired in our modern world-view with a willingness to change our misinterpretation of Nature, I present in this paper an analysis of ancient Chinese worldview opinions as they contrast with our present prevalent scientific assumptions. However, all this is quite fatally linked with adopting a true metaphysical knowledge about the real substance of the everlasting Multiverse of being (as well as of our Universe in it) and with our acceptance of its bipolar dialectical patterns of all existing processes within it. Modern science considers only a one-directional, especially linearly occurring fundamental axioms, such as entropy, laws of thermodynamics, or the law of conservation of energy. Ancient Chinese concept of the Great Change explains the Universe as a cyclical, infinite flow of contradictory dialectical transformations of its basic Energy, the Energy which is originally a Nothing/Emptiness, stemming from its antipodal bipolar forces=gravitation which is periodically (but not mechanically) reversing its direction. If its anonymous "Nothing" in the Multiverse/Universe (also in the being of our Earth) is not an absolute "nothing" but if it is that pure Energy, full of movement as well as full of information, permanently changing its antinomic polarizations, we are forced to consider such metaphysical
\end{abstract}

reality and synchronize our existence on Earth with its intrinsic laws.

Keywords Fundamental Energy (De), Nothing, Antipodal Bipolar Gravitation, Multiverse, Cyclical Metaphysical-material Coexistence, Law of Energy

\section{Introduction}

Our ability to renew and preserve favorable conditions for further survival of Humankind on Earth depends (in accordance with ancient Chinese philosophical wisdom) on man's perfect ability (as well as that of his family, tribe, nations and the whole population) to learn how to live correctly in an organically cooperative symbiosis with Nature, and thus really respect the laws of being, including in it also our respect to the natural needs of environments on our planet. Ancient Chinese came to this worldview on the basis of their verified evidence, obtained by perfect observations of natural processes and of the patterns of natural laws in their native environments, in which they continuously lived their peculiarly rural life style for many thousands of years. In doing so, they were convicted that the course of life on Earth is not a nonsensically chaotic process in which (only because of a quasi-absurdly-sudden and for no obvious reason) Mankind would be suddenly eradicated by a senseless disaster! A fortiori, if the ancient people in their basic lifestyles were ready to do everything to live in the symbiosis with the regular laws of Heavens/=Universe (i.e., according to the cosmic and natural regularities of that everlastingly changing organism of Earth's being) then each one of them would strive to fulfill his/her right role in the overall process of life within their rural communities of their innate human existence on Earth! In accordance with some ancient Chinese opinions, if one wanted to survive, he had to become a fully functional part of the natural duties of his 
family as well as of the whole common tribe of their Under-Heavens (下天) in the sense of a perfect harmony with the whole living organism of that countryside's environment! Similar observations are noticed now by modern natural scientists in their research of all natural forms of being, even though our modern Mankind (mainly due to currently very popular consumerist-hedonistic lifestyle and deriving from it the characteristic worldview) does not want to change bad habits and to adjust its terribly destructive relationship with the Earth.

\section{Is Our Modern Civilization Already Incorrigible or Is It Still Capable to Be Inspired by other Human Cultures for Better Relation with the Earth?}

It is true that millions of years ago some huge natural disasters abruptly ended the millions of years-long existence of dinosaurs on Earth, but in reality it was not at all just a random disaster but, at that time, a final period for one amazing chapter in Nature's realization on Earth, represented by a fantastic diversity of full spectrum and variety of dinosaurs' species, which would in their further transformations and possible mutations totally devastate the environment on Earth, and hardly achieve further enhancement in their evolution. Our Earth, in the process of its natural transformation was already awaiting a more interesting chapter in its development: - the emergence of mammals. This chapter could last, like that of the dinosaurs, also a very long time, if the most advanced species of mammals, the Humankind, would comply much more responsibly with the basic laws of Nature, and avoid devastating the functioning system of the whole Earth's organism. Unfortunately, modern Humankind continues to behave like a drunk who does not comprehend his irresponsibility, ignoring the proper human functioning in the body of the world! Moreover, much of the West has ideologically thrown away all the responsibility for life on Earth over to a transcendent "Somebody" and in the belief that $\mathrm{He}$ really exists, is sure that $\mathrm{He}$ would take care for all of it.

But by the time of the $20^{\text {th }}$ century, when sinology, alongside the other scientific areas, started its orderly development and deep scholarly research, one of its significant findings is the knowledge that for already 5,000 years at least two types of extremely contradictory human civilizations, with their significantly contradictory different ideological interpretations of the genesis of Humankind, have been developing on Earth in parallel: - thus not only the Western as the main one, but also the Far Eastern civilization (with China being from the ancient times its cradle and main ideological source!) among all other diverse populations living on the Earth.

While our Western civilization was formed originally from the nomadic Indo-European roots, and its European culture was in ancient times influenced mainly by the Greco-Roman conquerors' and militant lifestyle, the Far Eastern civilization, namely its original Chinese branch was already during the $4^{\text {th }}$ millennium BC transformed, (thanks to a special fully-built irrigation systems in the historical watersheds of the Yellow River, Yangtze Jiang, and later in the south also in the watershed of the Pearl River) into a profitable agricultural culture. This and the great geographical isolation from other world cultures during its ancient history caused that anything else than its original Chinese life style of the specially modified agricultural civilization was not attractive for them, and they have created within such life space a perfect symbiosis with Nature, without having any nomadic desires to conquer the world. Also later, in the Middle Ages, when Chinese were already some decades before Columbus circumnavigators and their fleets visited not only India and Africa but even some Middle and South American Indian empires, they did not yearn to conquer these countries and to enslave or plunder all those foreign empires. None of those far-distant countries was occupied by them, and also all along their history Chinese led, in the direction toward abroad, only defensive wars to ensure the security of the borders of their original territory. (Only for this reason they beset Vietnam, and some countries of Indochina, as well as they have subordinated in those times many of the adjacent nomadic neighbors to prevent them from attacking Chinese territory. For that reason in the $7^{\text {th }}$ century, before Tibet adopted Buddhism, they Sinicized the very warlike Tibetans and after the suppression of the An Lushan's revolt they pacified and in the year 840 in the northwest definitively subordinated also Uighurs, and in the south the originally barbarian tribes of Yue, together with all other nomadic tribes living in those $\operatorname{areas}^{1}$.)

Simply, the West and the Far East, especially China, are based on extremely contradictory civilizing characters from which emanated their very different lifestyles, which already from the very beginnings of their histories continually shaped their completely different ideological backbones of their outlook on the World. While from the Western perspective (even in the modern age yet for majority of people) Nature becomes only a looting terrain, the natural laws of which do not have to be respected by them, inhabitants of the Chinese "Kingdom of the Middle" had been through its history, together with their families and tribes, an organic part of the whole surrounding natural environment as it was their main nourisher and protector of their lives (e.g., in the traditional China all cities and towns were only the administrative centers for management of life and smooth running of the rural countryside). A local man in China was personally involved (in cooperation with natural

\footnotetext{
1 The ways and means of Chinese irrigation farming together with its agrarian social system and cultural habits has spread before the end of the $10^{\text {th }}$ century also to Vietnam, Laos and many other areas of Indochina. Also Taiwan, Japan and Korea were in this time very strongly impacted by Chinese cultural influences, even ideologically by their impact with Chinese Confucianism, Taoism, and Buddhism, which had very deeply influenced the religion and worldview of inhabitants of these countries.
} 
laws) in helping Nature to still better and better cultivate and revitalize the whole environment, of course, to develop there the most favorable agricultural conditions. Hence the whole natural landscape there continues to operate in its most optimal natural cycles. At the same time Chinese became existentially intertwined and vitally dependent on rural peasant surroundings, and thus functionally enmeshed with natural laws of those places. Well, while the Westerners in their human archetypes already from the time of Platonic-Aristotelian philosophical interpretation of the World used to explain the substance of being as a unitary metaphysical singularity set in its motion by Someone else, (e.g., a First Mover, or Judeo-Christian-Islamic monotheistic God-Yahweh-Allah), shamans in China already since the ancient times perfectly observed how is Nature functioning, and philosophers some century later (mainly their first one, Laozi,) began to understand, why and how is everything created by the contrarily synergistic movements of its transformations. Those primeval shamans' observations inspired a collection of practical advices for people on how to properly and in the best participation with metamorphoses of Nature manage their longest and undaunted survival. As a mystic relic from those earliest beginnings of Chinese cultural heritage, there is the Book of Changes (Yi Jing) [1] which has been used till nowadays by many people also from the West for consulting their special life situations.

In the middle of the $5^{\text {th }}$ century $\mathrm{BC}$ all the Chinese shamanic knowledge had been reinterpreted into a complete metaphysical, philosophically-anthropological and ethical system by one of the greatest Chinese philosophical synthesizer, the Old Master (Laozi). In contrast to our Western monotheistic and subject-object's philosophical interpretation, he explained the basic substance of being absolutely non-theistically as an organically vivid unity of an endlessly continual circulation of yin-yang(=antipodal) dialectical processes of transformations, realized by its immanent Way (Tao, Dao 道) of an anonymous and bipolar dialectical Energy (Te, De 德) of Nothing (or as its Emptiness). However, thanks to all needed knowledge and information encoded in it, it operates inside the Universe (= Heaven 天) any and all what is necessary to happen. Thus in every one of its particular space-times there does not begins and exists any form of being as an absolutely static singularity but basically only as a process of its changes! Still and everywhere within the interior hub, tensions of the antipodal (in Chinese: yin-yang) contradictions flood this bipolar substrate of the vacuum Energy of Nothing, in which due to its endlessly antithetical polarity all material forms and things as well as any spiritual entities of this time-space's world are created, exist and become extinct, alternatively changing inside its bipolar-antipodal polarity into still different and different forms (entities) and structures. Chinese called these cosmic transformations $a$ Great Change (大易). (In such sense also our living reality is similarly alternative, and nothing in it is caused miraculously and arbitrarily by any lasting singularity of goodness or by a specific transcendental quality!) The Great Change, according to Laozi's (and later by many other Chinese metaphysical theories of being) is happening in infinite varieties, self-organically in itself organized anonymously by all the needed knowledge and information encoded in its basic Energy of being!

\section{How Could This Classical Chinese Theory of Being inspire Modern Scientists?}

The Westerners as well as the Far Easterners live on the same planet, and thus if the civilization at one side of the Earth argues about the substance of being as something completely different than that one from the other side of the World then the real truth about it has to be only one of them.

Almost until the end of the $20^{\text {th }}$ century the Westerners not only religiously but also in their worldview and philosophy were convinced, mainly thanks to Hegel's Phenomenology of Spirit (Phänomenologie des Geistes) and his Lectures on the Philosophy of World History (Philosophie der Geschichte) [2] which assumes an idea of a linear and subject-object causality of the creation of being by a leader "Thesis" or a "Creator", that any other truth than that one cannot exist. This conviction was strengthened also by the well-established Christian dogmas of the apocalyptic truth about an existence of a transcendent God-Creator-the First Mover and about a creation of the Universe by Him as initially a motionless being which He created and enlivened in an absolute "nothingness". But today against such "truth" we have already inalienable, metaphysically nonetheless justified truth about the substance of being of the Far Easterners (originally of Laozi's thoughts) according to which there is no transcendental Subject as a First Mover or a Creator of being, because the basic substance of $\infty$ Multiverse/Universe of being (Heavens=Nature) is eternally existing in constantly whirling cycles of creations, existences and extinctions of an endless sum of material - spiritual structures and beings, forms and things, proceeding from the oppositely polarized antithesis of an anonymous pure Energy (Te, De) through Its immanent Way (Tao, Dao) of an everlasting process of being. It includes in itself inside such immanent processes of this Energy all necessary information, knowledge and wisdom needed for the Way (Tao, Dao) of creating anyone reality without a need for any transcendent God-Creator or a Subject of its dialectical transformations. Whole $\infty$ Multiverse, which also includes inside it our time-spaced Universe, has in itself (thanks to this Energy) everything what it needs for its duration, and thus is always ready to change into all various forms of its multitudes of organic as well as inorganic life.

An expectancy of such Far Eastern (especially Chinese) metaphysical truth had been noticed already by G. W. Leibnitz, inspired by Chinese knowledge about yin-yang (=antipodal) dialectics, graphically expressed by bagua triagrams (八卦) [3]: 


\section{1. $\equiv \equiv \equiv \equiv 000000$ \\ 2. $\equiv \equiv \equiv \equiv 001001$ \\ 3. $\Xi \Xi \equiv \Xi 010010$ \\ 4. $\equiv 011011$ \\ 5. $\equiv \equiv \equiv \equiv 100100$ \\ 6. $\equiv 101101$ \\ 7. $\overline{\equiv \equiv} 110111$ \\ 8. $\bar{\equiv} \bar{\equiv} 111110$}

His concept of the "Monad", as the basic substance of being represented in itself the reality as a complete concept containing all predicates of an endless self-organized subject of changing inside it, was inspired there by this Chinese metaphysical truth. From his correspondence with the French missionaries in China (namely with de Bosset) [3] it is clear that he was inspired by the information received from them about the Chinese vision of the processes in Cosmos in such organically, antipodally changeable and thus (in Chinese) yin-yangly movable sense:

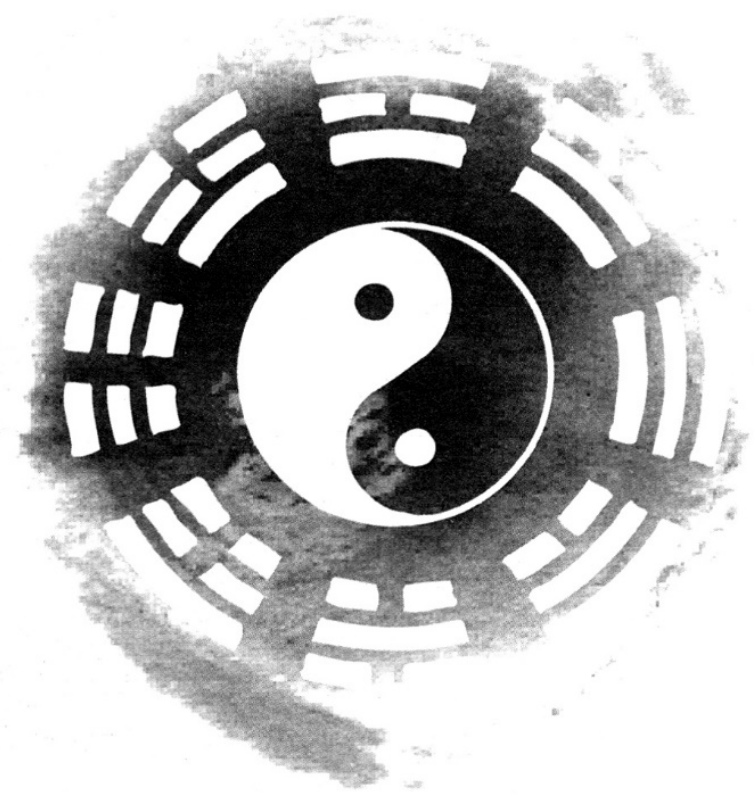

Also in his correspondence with the natural scientist Papean, Leibnitz accepted the basic truth that the sum of energy passes through things without omission or without any reduction of its total quantum [3]. He thus confirmed by all of it that the world is a vivid unit in which things at their boundaries are able to forward their energy, (e.g., thermal energy) without any loss in its total universal or cosmic quantity. And if it is acceptable for our Earth and Cosmos, the same truth could be relevant also for the whole everlasting Universe/Multiverse of being. 
On one such principle are based and defined by Western natural sciences also the Laws of Thermodynamics, (especially the $2^{\text {nd }}$ one) which define "the conservation of energy"[4]. Of course, it is a version of the law of conservation of energy, adapted only for thermodynamic systems, but its fundamental truth is, that the total energy of an isolated system is constant; energy can be transformed from one form of being to another, but no new one can be created or destroyed, it only changes in its different forms. [4] Well, if we accept also the endless Multiverse of being as an isolated system of its endless inherent totality " $\infty$ ", then the Laws of Thermodynamics could be only subsets of the general Law of Energy unto Itself. It ought to proclaim:

\section{Pure E $(\mathrm{Te}, \mathrm{De})=\underline{\text { by Its anti-poles infinitively active }}$ Nothing, \\ An immanency of Vacuum full of all needed information for an endlessly cyclic antipodal emanation of changes of its bipolar dialectical self-realization of a Multiverse/Universe of being}

$$
=+\infty-
$$

And when we acknowledge that Energy unto itself ${ }^{2}$ is not any form of a material substance but it is in itself the most fundamental and (thanks to its polarity) the most self-movable substance/substrate of the Multiverse/Universe/Cosmoses of being, and is known by us as Vacuum, thus its basic quantity there is constant, (regardless of its various space-time's forms creating, existing and changing in any specific space-time reality) and cannot be as sole One created or destroyed at all! It lasts infinitively thanks to circulation inside its opposite poles of charge.

And what further follows when this basic pure Energy will transform into a concrete space-time form of itself, was defined by Western natural science in the Second Law of Thermodynamics [4]. Western physicists understood it with the help of experiments with the empirically most abundant form of energy which is thermal energy. According to the Second Law of Thermodynamics the thermal energy is transferred (or transformed) always in one direction, from the more heated medium into the colder ones and during this process it has a natural tendency in every space-time's isolated system to slough off and degenerate into still larger quantity of smaller and more disorderly states and fragments of that system. After discovery of this entropic tendency of thermal energy in every isolated reality, and applying it to our whole Cosmos as its own isolated system, it starts to be clear that all its components which send forth heat are interconnected by the process of entropy, e.g.: for Ludwig Boltzman it was a very depressive discovery from which he's drawn a conclusion that, consequently, our world has a

\footnotetext{
${ }^{2}$ The substance of the fundamental Energy (De) of being is forever movable Nothing/Vacuum, thanks to its bipolar positively-negatively (yin-yang) everlasting polarized medium which can never be in a state of a static singularity.
}

tendency toward its definitive disintegration. Also Saibal Mitra stated, in accordance with a similar discovery, that at a very microscopic level it simply states that if we have a system that is isolated, every natural process in that system progresses in the direction of an increasing disorder, or entropy of the system, which also means that the Cosmos cools down because the same finite quantity of the thermal energy is dissipated into an ever increasing quantity of atoms $(\mathrm{S}=\mathrm{k} \log \mathrm{h})$. Theoretically it means that the chaos and disintegration are increasing more than any progression to a constant order. Even when order is increased in a specific location, for example by the self-assembly of molecules to form a living organism, there is always a net increase in entropy, and thus the process of the World, taken as a whole, results in a net increase into the disorder. Of course, this is quite terrible discovery, because it confirms that our whole reality is in progression from order to chaos, and thus chaos is the ultimate fate of everything in the future of the World! If it is so and the entropy is a measure of chaos, the total energy in Cosmos would be in a permanent process of disintegration and destruction, making everything here progressively worse and worse! But is it truly the tendency in our Cosmos as well as in our World (taken as an isolated subsystem) of only the one directional and thus only the linear movement of entropy, from one more heated source into many smaller ones ever colder and colder? Didn't our Western physicists make a fatal mistake in their logical conclusion from such discoveries? In my opinion, they made the mistake because they have considered only one linear directional movement in the reality of things in our world, from order to chaos (taking into account only the behavior of thermal energy in Cosmos within its space-time material structures!)

In accordance with ancient Chinese metaphysics, the Heaven $=$ Universe $/$ Nature or better the whole Multiverse of being follows the antipodal (=yin-yang) cyclic process of a Great Change which "never overruns its edges" [5]. As the ancient Chinese philosophers noticed (especially the first one, Master Laozi) in Nature (and thus in Heavens as well as in our world) everything moves from one extremely marginal position in one direction to dialectically contrary events, processes, and entities, turning at once to the totally reverse tendencies and qualities. The truth about it is philosophically proclaimed, for example in the text of Huang-Lao ${ }^{3}$ : " $a$ perpetual activity is its most characteristic feature and Harmony is its function!" [6] And thus from the metaphysical point of view its basic characteristic is primarily this one:

\footnotetext{
${ }^{3}$ Citations are translated by author of this manuscript from Chinese original of the $4^{\text {th }}$ book of Huang-Lao si jing, ed. by Chang L. S. and Yu Feng (1998), published in her monograph, titled Laozi and the Process of the Genesis of Dao De jing (in Slovak), $2^{\text {nd }}$ Vol., Bratislava, Veda Publishers 2012, pp.258-9.
} 


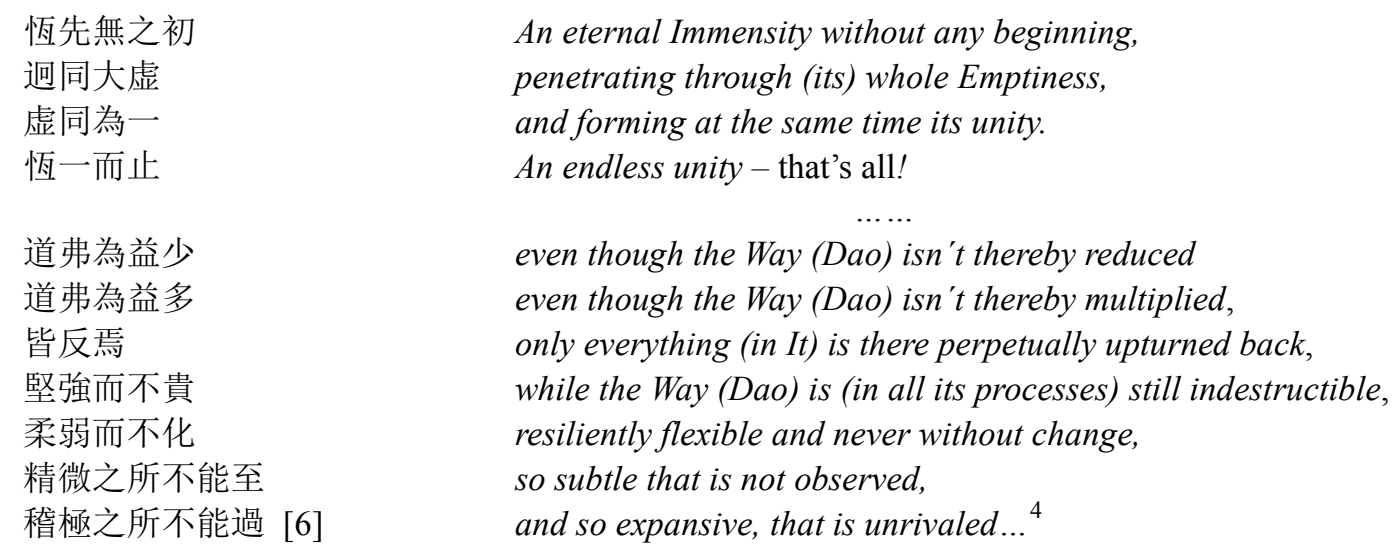

Such is, for example, one of ancient Chinese general definitions of the pure Energy in its everlasting movement, which is the basic substance and the whole total substrate of being and from which everything in the endless ( $\infty)$ Multiverse of being is created as well as into which everything is also absorbed in its perpetually antipodal circulation. Our modern natural scientists discovered by their quantum physics research as well as by accelerator experiments, that this Energy can stream at once in the form of a pure boson as well as in the forms of the time-spaced atoms and anti-atoms, electrons and anti-electrons, matter and anti-matter, but I am afraid, they are making one fatal mistake, because they accept in it only the behaviors of its material forms (atoms, electrons and time-space matter) as well as only their thermodynamic form of energy for defining the laws of Universe, generalizing them for everything everywhere, and at the same time not fully researching the behaviors of its anti-atoms, anti-electrons, anti-matter or the boson counter-processuality. Of course, in such case they have been considering only the laws of behaviors of the mass of matter and not the laws of behaviors of anti-matter, as well as of atoms and not also the laws of behaviors of anti-atoms, and thus only with the stream, for example, of thermal energy from its heat source of mass to the colder parts and not at the same time also with the nature and laws of antithetic streams of the anti-mass to its "boson's" substrate or to the most fundamental entity of the pure Energy! Yes, empirically we can discover that the streams of atoms in the Universe have their character of entropy but because there exist also anti-atoms' and anti-matter's streams, (confirmed already with scientific discoveries that they presumably exist!) we would have at the same time to take into account an equally importance of the laws of these opposite flows! And in such case, of course logically, not visibly, to take into account that the entropy out there ought to change metaphysically into a new reverse tendency, and thus such forms of Energy once again return to the new or some different space-time's entities of creation of being. Thanks to these reverse circulations of anti-atoms or anti-mass inside the Multiverse there, quantum energy creates in the concrete space-times a new visible mass of atoms, again and again creating newly visible worlds. Thus the entropy also in our Cosmos ought to be only one part of the complete process of a perpetually contradictory antipodal circulation of being. Paul Dirac [7] anticipated something about it already in 1928 when he noticed that the mass of matter can come into existence from vacuum and later again to disappear into it. He tried to express it by the following mathematical equations [7]:

$$
\begin{aligned}
\gamma^{0} & =\left(\begin{array}{llll}
0 & 0 & 1 & 0 \\
0 & 0 & 0 & 1 \\
1 & 0 & 0 & 0 \\
0 & 1 & 0 & 0
\end{array}\right), \gamma^{1}=\left(\begin{array}{rrrr}
0 & 0 & 0 & -1 \\
0 & 0 & -1 & 0 \\
0 & 1 & 0 & 0 \\
1 & 0 & 0 & 0
\end{array}\right), \\
\gamma^{2} & =\left(\begin{array}{rrrr}
0 & 0 & 0 & i \\
0 & 0 & -i & 0 \\
0 & -i & 0 & 0 \\
i & 0 & 0 & 0
\end{array}\right), \gamma^{3}=\left(\begin{array}{rrrr}
0 & 0 & -1 & 0 \\
0 & 0 & 0 & 1 \\
1 & 0 & 0 & 0 \\
0 & -1 & 0 & 0
\end{array}\right) .
\end{aligned}
$$

\footnotetext{
${ }^{4}$ See in Dao Yuan in Huang-Lao xi jing, ed. by Chang L. S. and Yu Feng (1998); or by Chen Guying (1995); as well as in Chen Yaoting's edition from Shanghai Academy of Social Sciences or The Taoist canon under the title: Zangwai daoshu, compiled by Hu Daojing. Bian zhu (a collective of compilers) in: Mawangdui han mubo shu, Wenwu chuban shi, Beijing 1980. English translation by author of this manuscript.
} 
Thanks to him, natural scientists began to consider an anticipation or a suspicion that all things are able to change their mass into its opposite, similarly as an atom is able to coexist inside the system of its being with the anti-atom, electron with an anti-electron, and they are only two examples of parts of the whole cycle of all antipodal parts of the complete changeability of the reality of being. All these processes in their equilibrium in one direction are able to change into its exactly reverse quality with the tendency of flow as well as transforming into their reverse forms of existence. In coherence with this truth anything in the world would not be only in the state of its linear process of entropy, as in the everlasting Multiverse of Energy (and thus also in the basic substance of the current cycle of Nature of Earth) it is impossible, in accordance with the discovery of those reverse tendencies, that only one side of such circulations or only linear and entropic process could be its complete reality. Everything in this reality is inferior to the tendency of the dialectic turns from one marginal position of one direction of being into the reverse tendency of its opposite existence, accordingly to the law: "never overruns its edges". All the more we have to accept this truth after Max Plank's discovery of an attribute of elementary particles, from which it is clear that although in vacuum there is an infinitely vast bulk of atoms as well as quantum particles which are moving there at maximum speeds, everything is moving in its own direction. From the outside it looks like an absolute chaos, but in reality it is a perfect order without any possibility of a collision. That "chaos" is in its metaphysical reality a perfect net sum of circular courses of atoms reversing into anti-atoms, and quantum particles reversing into those with an opposite charge. However, from all these circulations we are able empirically to discover only the tendencies and laws of that physical and empirically perceivable part of the circle, not of the opposite one. A pure Energy (Te, De) is not missing in the Universe, despite those for appearance's sake quasi entropies and thus the Multiverse of being is not in the process of Its cooling, although it is true that the flow of the time-space thermal energy is advancing in only one physical direction from the heat source to any still colder and colder parts of being. In its (meta) or anti-physical direction it always turns with their own patterns of circulation to its opposite form, thus completing the indestructible Great Change of the pure Energy in its ceaseless antipodal dialectical movement of its perpetual turnovers.

For that reason the present Laws of Thermodynamics do not represent the whole truth about the complete laws of the reality of being as they identify only a half of the overall truth, that one which is identifiable from the point of view of physic but not the one from another side of the complete structure of natural processes of being. One of further observation of this truth could be confirmed also by Heisenberg's Uncertainty principle [8]. It says that there is fuzziness in Nature, a fundamental limit to what we can know about the behavior of quantum particles and, therefore, the smallest scales of Nature. We cannot measure the position (x) and the momentum (p) of a particle with absolute precision. The more accurately we know one of these values, the less accurately we know the other. Their Great Change in the Multiverse/Universe of being is not any mechanical net of movements but a highly sophisticated structure like of a vivid organism, where quantum particles being created there from vacuum in unpredictable ways and without any Subject's determination and vice-versa, they at once change from the matter of things into the pure energy, without anybody's verdict or decision.

According to the Laws of thermodynamics, as defined by our modern physicists everything in a concrete vacuum is realized mechanically by borrowing its energy from the more heated source to a colder ones, in which process its initial sum crumble and crumble into more and more fragments, causing the whole Universe undergo the process of entropy. But if we agree that our Cosmos isn't the space only of atoms, electrons and matter but also the space of anti-atoms, anti-electrons and anti-matter, then this other side of its reality must have, of course, also its own quality of being and special laws of movement, which guard in the opposite cycles the return of all that is "dissipated" by entropy to the new starting position of the recurring heating source. Only that can ensure normal circulation of energy inside the endless Multiverse and its multi-various forms of being as observed already 2,500 years ago by Master Laozi:
無為為之而合乎生死 無為言之而通乎德
Without doing anything, everything is done, granting the destiny of births and dying, without (any explanation) of it in words, everything is then flowing back into the pure Energy (De),
恬愉無矝而得乎和 有萬不同而便乎生 in a settlement of satisfaction, receiving its harmony and quietness without any regret, into ten thousands of quite different multiplicities transforming (and remodeling at once) again and again into life
和陰陽...[6] in the mutual dialectics of yin-yang contradictions.... 5

With such understanding, we could express a new $2^{\text {nd }}$ Law of Energy (as the basic substrate of being) by this definition:

\section{basic pure $\mathrm{E}=$ endless ability of doing (everything) by bipolar multiversal dialectical contradictions inside a highly organized changeability, without any pre-directed determination}

\footnotetext{
${ }^{5}$ Citation is translated by author of this manuscript from Chinese text of Dao Yuan in Huang-Lao xi jing, ed. by Chang L. S. and Yu Feng (1998);
} 
This Energy, perhaps, could be interpreted (at a first glance) as something mysterious, even as a self-sufficient Mover or a God, able of all subjective decisions, but contrary to such interpretation all processes of this Energy are realized immanently within it but without it is able to make any subjective decisions, as if this Energy was "a slave" of the dialectical regularities without any self-subjective interventions influencing them, and without any own subjective consciousness of their projections! Thus there cannot be any subjective Mover or a Creator (as a conscious singularity) controlling them! In accordance with Chinese graphical expression of this process we are forced to understand it only like an anonymous yet still regularly (though at first glance looking like a total chaos) changeable bipolar dialectical process, graphically expressed by the 64 yin-yang (or even more clearly by its 128 antipodal) hexagrams:

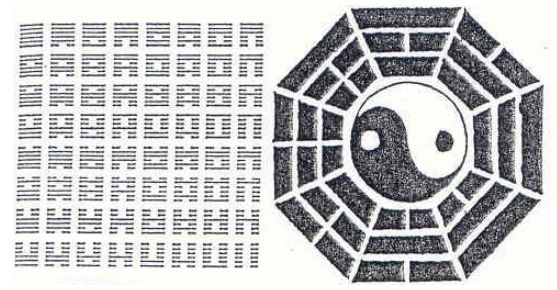

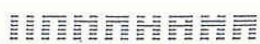

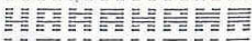

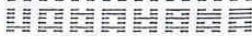

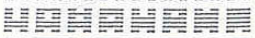

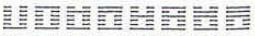

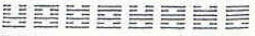

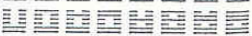

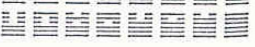

For a better understanding of these graphical expressions one needs to perceive every one of these hexagrams not as a static symbol but as a progression (or a regression) at once of its own forever regularly changeable movements. All together it expresses in every sense and in all directions quasi a moving chaos of the uncountable multitude of highly various cyclic movements which are at every moment absolutely different one from another. Really, we could interpret it as similar to the modern idea of "strings" which are something like the above graphical expressions of the directions of movements of photons and elementary particles in vacuum inside their very chaotically speeding movements, but contrary to their visualization, these antipodal (in Chinese: yin-yang) dialectical dynamics have their integrated cyclic directions, where each one exists in an gradually organic coexistence or contradiction with another. Thus this (for appearance's sake) chaos is (as you can see in its graphical expression above!) as a matter of fact a highly organized order. All its bipolar dialectical processes are in their complete sum again and again in one direction toward entropy but at once reversed in the opposite direction toward a reintegration of its pure energy to the new creative metamorphoses in accordance with the truth that the energy of each isolated system is constant.

\section{Potential Consequences of Such Metaphysical Discoveries on Changing our Worldview Orientation}

Acceptance of an anonymous and bipolarly active Energy which is without any own subjective self-consciousness but which is in spite of it a dialectically self-movable basic substance of an endless Multiverse of being could, of course, radically change our traditional Truth about the origin of the World. Here in the West, for centuries we have persuaded ourselves in our everyday life to the "divinely revealed Truth" according to which the world was created by a divine act of the transcendent God who created it as an object by which $\mathrm{He}$ would be conscious of Himself. In the metaphysical sense of such Truth the Western metaphysics seek to interpret it philosophically by a linearly dialectical model of Subject-object causality, in which the active One is the creative "thesis" in the starting proposition as the only active Subject, and his dialectical antithesis is an object, created by Him, in which this active Subject-proposition would be able to self-realization of Himself by becoming His own self-consciousness. Such dialectical antithetical object has in this type of being only a passive function in which the active legitimacy belongs only to that One/Subject and so He, as the only active Subject, has the authority to determinate its/their fate/s.

Mankind is in the ethical plane of such concept in this particular world only an image of God and functionally people thus ought to be only His objective "theses" in the living processes of our terrestrial world. But as such they are designated and so forced to accept in every one of their activities the divine laws of God and to realize in the world a quality of goodness of His divine ethical conception. In opposite case they would be "evil", given for liquidation. In such Western interpretation man is authorized by God "to struggle with the opposite" as with "evil" for its total liquidation. And thus people in the monotheistic Western culture have pursued already for centuries a world-view that the life is not about harmony and cooperation of opposites but about the "struggle" of one opposite with another, dreaming about the final victory of the first one over the second one. From this point of view such is their duty stemming from the active thesis, representing goodness, over the opposite pole "representing" of course the evil! In the ethical plane it forced people, for example to keep a very cruel search among them for opposites who, if considered "evil" have to be singled out for their rectification or liquidation.

Could you imagine such a goal or process of total liquidation of opposites in the basic structures of things in our being?! For example a total liquidation of a negative charge of anti-pole in the structure of atom or of anything what the antipodal process of the Great Change's basic Energy (De) in the life reality of Universe represents?! It is impossible to have in the structure of atom only the positive $(+)$ polarization without its negative (-) one, doesn't it?! Since the basic laws of life are the same everywhere in the structure of Universe, thus it must be valid forever also from the ethical point of view that there cannot be anybody authorized in our terrestrial reality to believe in the righteousness of such one-sided, unbalanced principle of existence in the living processes of our social reality! But people, thanks to such skewed worldview, after adopting the 
monotheistic religions (or some similar political ideologies) began to enact it, for example, also as the need for "the struggle", and thus for the need of total liquidation in our social reality of pagans or other people with differently oriented ideology or faith. Thus all who are not practitioners of the right belief or of the right ideology became to them enemies whom it is moral to destroy in this life's reality.

Today, however, when Humankind is becoming a global closed system, such world-view could be a very dangerous leading case, and as we already experience, is able even to justify acts of terrorism by some. Well, what about letting us to be inspired with the ancient Chinese antipodal (=yin-yang) dialectical metaphysics of harmonic coexistence of anti-poles instead of "struggling" for their total liquidation?!

At the turn of the $6^{\text {th }}$ and $5^{\text {th }}$ centuries BC., a genial Chinese thinker Laozi (the Old Master), living in the not-yet-devastated Nature and spending a large part of his life as an archival collector wandering for many years in all corners of China's countryside, perfectly observed and understood the basic laws of natural processes of Tian (Heavens=Universe as well as the whole endless Multiverse of being) and derived from it some fundamental deductions about the truths of life on Earth. He expressed it philosophically mainly in his work, which the World knows today as Dao De jing. (The very fact that his philosophical work to these days is generally accepted not only in China and is among the most translated Chinese books in the world, says it all!)

Well, what is it that (contrary to our western philosophers) Laozi observed metaphysically highly correctly from Nature and from the laws of our Cosmos' reality already in the $5^{\text {th }}$ century BC? His most important discovery was that our whole World is only one of the quantity of the concrete visible forms of the basic Energy (De) of Heavens=endless Multiverse and Cosmoses of being, and the fundamental nature of this Energy is an antipodal (=yin-yang) polarization and a bipolar dialectical movement between these its anti-poles, which makes it a self-moving process in all its forms, structures and creations. Creating all matter (thus also our particular space-time being) through such dialectic process which is conditioned by its anti-poles' functional counterparts, they are from the point of value equivalent one to other and none of them is less important or more "immoral" than the other. Thus neither of them should be designated for liquidation before its "overflowing the measure"! (Today we already know very well that as any kind of energy cannot be created from just one (let's say) only "positive" charge, neither life, which is a spatiotemporal manifestation of that basic substance of being, cannot be run only under the positive, in Chinese: "yang", existence of itself, too!) Thus there is no reason for an absolute liquidation of anything only because of its opposite quality within the process of polarization. In an anonymously structured organism of the endless Multiverse of being every nascent particularity (also in our Cosmos's and Earth's space-time) is functional only within the existence of its antipodal processes of polarization and thus everything exists in their specific form only for as long as they have there the real inverse justification and as long as they fulfill in their cycles of transformation their true respective function, of course always adhering with the contrary (in Chinese: yin-yang) tension of its basic nature! This is reflected even in the basic structure of the smallest particle of our material world, "atom", which is a synergistic existence of inseparable positive (protons') and negative (electrons') charges in it! However, the fundamental truth about such theory of being was philosophically discovered already by Laozi in those ancient times in China!

Traditional Chinese cultural consciousness from the beginning formed in itself the Taoist-Confucian worldview, incorporating into its faith the need for a perfect cooperation of Humankind with Heavens and Earth. Such idea was embodied into a perfect impersonator of it, the Sovereign 王 (Wang) who would be ready to coordinate all social processes with the laws of Heavens 天 (Tian=the whole space of Universe) and all processes on Earth 地 (di=the whole terrestrial space of living beings) into the perfectly cooperating Harmony of contradictions. The highest possible moral quality for such coordinator had been embodied there into the paradigm of "sheng ren 聖 人”, what means a Sage or the Real Man in his entirety, not only in personal traits but also the most perfect Humankind's identity representing the highest quality of all human beings. But in accordance with that philosophical conception such representative of Humankind neither ought to be a "good" man (in our Western ethical sense) but first of all (because of his paramount function) the most perfect manager and relentless coordinator of all social processes in the tangible reality between Heavens and Earth to make them correctly functional in every type of cooperation and organic synergy of Humankind with cosmic and earthly-natural forces of being. For that reason, as Laozi says in Dao De Jing, the Real Man (Shang Ren) being the highest representative of Humankind in the World, in some single realities of life should deal with people as with "the jackstraws dogs" [9]. It is because social processes on Earth are in their bipolar antipodal functional reality on the same level as the natural ones, and thus he cannot be in every case a "good" man (in the sense, for example, of Christian acceptance of "good" moral quality), but to the contrary such manager of Humankind ought to operate in accordance with the both antipodal polarities, similarly as that traditional Chinese concept of behaviors of the legendary Chinese Emperors, Yao, Shun and Yu:

\footnotetext{
${ }^{6}$ The straw dogs were in ancient China used at the spring holidays' rituals for scaring some bad demons and after completing these ceremonies they were burned down as no longer necessary. This citation is from: Laozi Dao de jing, $5^{\text {th }}$ chapter, see: LAOZI zong mu he Wang Bizhu in the book LAOZI, ZHUANGZI, No. 9213 of Su-pu pei-yao, Chung-hua shu chu, Taipei 1965 (reprint)
} 


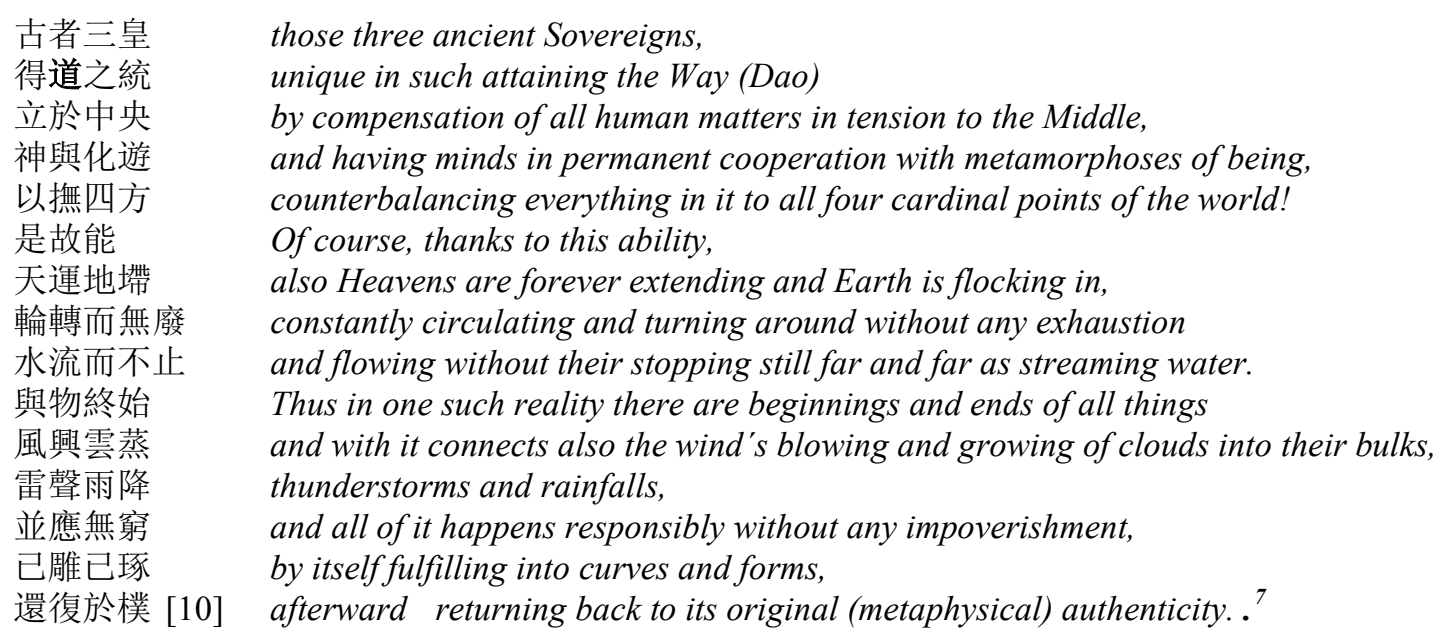

As we see, here is quite another description of the Perfect Man (Shang ren) than in the sense of our Western moral qualities and positive ethical ideals of Christian Humanity! The description of the best(=perfect) Man and, for that reason, an ideal sovereign is foremost to be the personification and thus a respectable representative of the perfect coordination of all social processes into their concurrently synergic functions with Nature in their contrary-dialectical (=yin-yang) moving reality between Heavens and Earth. And thus not only "goodness" or an "evil" of some people are in the center of interests of the Perfect Man in such World's reality, but mainly his overseeing the right functions of all different people in the completely coordinated social organism of the endlessly changeable reality of the Great Change among the Heavens and Earth. And such reality, of course, can never be only "good" or "evil" and thus cannot be dictated to everybody in the sense of a total victory of goodness over the evil, or contrariwise. The correct function and purpose of our life, and thus mainly that of our sovereigns and political managers in the concrete reality of the world isn't in legitimizing to uproot "an evil" or "to fight with the contrariety", but to make everything possible for the best harmonization of all the positive and negative processes between their anti-poles in the sense of not overflowing the reasonable measure in any of them, and thus not in anything "overruns its edges". In accordance with the Book of Great Change [1] vice-versa in the following sense

\begin{tabular}{|c|c|c|}
\hline \multirow{24}{*}{ 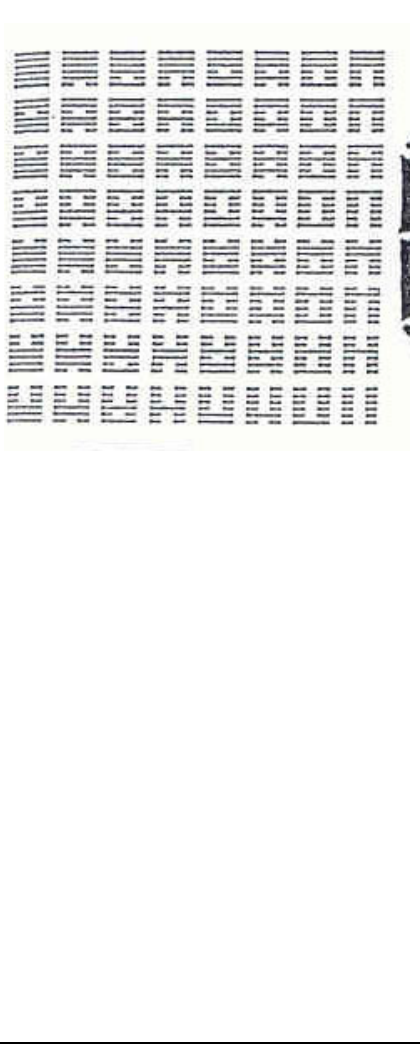 } & 111111111111111111111111 & 000000000000000000000000 \\
\hline & 111111111111111111111111 & 111111111111111111111111 \\
\hline & 111111111111111111111111 & 111111111111111111111111 \\
\hline & 111111111111000000000000 & 111111111111000000000000 \\
\hline & 111111000000111111000000 & 111111000000111111000000 \\
\hline & 111000111000111000111000 & 111000111000111000111000 \\
\hline & 111111111111111111111111 & 000000000000000000000000 \\
\hline & 111111111111111111111111 & 111111111111111111111111 \\
\hline & 000000000000000000000000 & 000000000000000000000000 \\
\hline & 111111111111000000000000 & 111111111111000000000000 \\
\hline & 111111000000111111000000 & 111111000000111111000000 \\
\hline & 111000111000111000111000 & 111000111000111000111000 \\
\hline & 111111111111111111111111 & 000000000000000000000000 \\
\hline & 000000000000000000000000 & 000000000000000000000000 \\
\hline & 111111111111111111111111 & 111111111111111111111111 \\
\hline & 111111111111000000000000 & 111111111111000000000000 \\
\hline & 111111000000111111000000 & 111111000000111111000000 \\
\hline & 111000111000111000111000 & 111000111000111000111000 \\
\hline & 111111111111111111111111 & 000000000000000000000000 \\
\hline & 000000000000000000000000 & 000000000000000000000000 \\
\hline & 000000000000000000000000 & 000000000000000000000000 \\
\hline & 111111111111000000000000 & 111111111111000000000000 \\
\hline & 111111000000111111000000 & 111111000000111111000000 \\
\hline & 111000111000111000111000 & 111000111000111000111000 \\
\hline
\end{tabular}

\footnotetext{
${ }^{7}$ English translation by the author of this manuscript from Chinese text of WENZI: Wenzi zuanyi, citation from the $1^{\text {st }}$ chapter in No. 9221 of Su-pu pei-yao, Chung-hua shu chu, Taipei 1965 (reprint).
} 


\begin{abstract}
$+\infty-$
As the reality of being in the Multiverse/Universe/Cosmoses as well as in our world on Earth (because the endless totality of its basic Energy De) has just such character! Also Humankind on the Earth can survive only if we adhere to these dialectical laws of its reality and will not "overflow the edges" of the life possibility. In other case the nature of this basic Energy automatically starts on Earth some partial or more extensive processes of our liquidation.
\end{abstract}

\section{Conclusions}

That what was already 2,500 years ago hypothetically expressed by the Old Master (Laozi) in his work, is being confirmed today in scientific conclusions (though often very reluctantly) by modern natural scientists and by their most advanced latest experiments. Particularly the most recent astrophysical and quantum theories and above all accelerator physicists try to get deeper and deeper into the "bottom" of the substance of being of all material matters. In doing so they exceed already the boundaries of material structures and in an organism of all containing reality they find everywhere and in everything a being of antithetical (in Chinese: yin-yang) synergy of positive and negative charges in the tangible as well as intangible components of being, which in one or another way exist everywhere in the world. In such experiments scientists also approach something far beyond the masses of material forms, something what is already in the realm of a sheer energy of being.

Now, when there are many television documentaries ${ }^{8}$ on the subject, based on all those experiments, even an ordinary layman is already aware that our Cosmos started and till now lasts inside an endless Multiverse of being and is just one of numbers of its immanent "singularities(?)" (Containing in their basic Energy all needed information of its anonymous wisdom). But that Multiverse of being is forever and never had its first moment. Yet the authors of modern TV documentaries admit that time is just only an auxiliary dimension of the cosmic space where everything nascent, exists and disappears in circulation of the total variety of forms and possibilities of being. Well, and the Multiverse of being, because of its bipolar basic Energy metaphysically is an endlessly moving infinity, it isn't any concrete singularity, and as such it didn't need any divine intervention that would make it come "to be"! That admits even the famous "hunter of singularities", astrophysicist Stephen Hawking [11], according to whom the matter, as well as space and time are crushed out from the substance of being by a Big Bang, whereby our Big Bang is not the beginning of an existence of the Multiverse but only one episode within it, initiating the formation of just our present Cosmos which at once will end

\footnotetext{
${ }^{8}$ For example I refer here to some discoveries publicized in several television astrophysical documentaries, broadcast on BBC and Spectrum Chanel in years $2013-15$.
}

up in a black hole or in a shrinkage (similarly as our sun becomes a so-called dwarf). But the Multiverse of being in its pure Energy will exist there forever, creating infinite number of other Big Bangs initiated by its own inner forces that will result in still new and different singularities of forms and structures of being. Consequently this also means that there never existed anything absolutely and actually empty, what monotheistic theologians preach as a nothing inside which God would create being, but that there exists a completely different way of "Nothing" full of the constant, anonymous pure Energy kept in the hub of its antipodal fields that is at once the medium of all information needed for all evolutions and self-creations of all the realities inside it. It is the ubiquitous substrate of the entire circulation within the whole infinite being of the Multiverse. This super-Energy is from the material point of view a vacuum of "emptiness", though in reality it is full of dialectical motion and frequency of movements, but in its normal state cannot be reflected or visualized in our space-time reality. (The scientists managed to detect its manifestation recently only for a split moment by the CERN accelerator!) Thus in the modern interpretation of astrophysicists it is, therefore, a quasi-vacuum, visualized in the Universe as a Darkness. Because of such inability of detection, modern quantum science and astrophysics started to use for it quite frequently also the concept of a Dark Energy. And although scientists still do not know precisely how to define this energy, it became already a scientifically proven part of the constant content of the Universe.

However, because the infinite Multiverse of being is formed, managed and organized in itself by this pure substrate (for us looking as Dark or invisible Energy) through its own naturally internal and antipodal movable code of being, and thus not by an externally transcendent God or its own in itself existing Subject, thus our idea of God (whose existence had not been questioned nearly to the modern times!) logically becomes now, thanks to all these discoveries, an outmoded paradigm. Thus it is already scientifically confirmed that not only in our spatial reality but in the $\infty$ Multiverse of being, there is no place for God. Mainly because our Universe too, as one of the multitude of forms of being, grew about 13 billion years ago in the process of transformations (or Big Bangs) within the infinite Multiverse from this anonymous Energy, thanks to its permanent contradicting polarity of natural dialectics made possible by its nature and as a kind of a multi-cosmic code anonymously operated with its immanent information. Ostensibly it looks as a perpetually changing "chaos", but on the contrary inside itself (maybe thanks its immanently organic regularities) it is a highly sophisticated order! Thus it is not a chaos of the totally catastrophic destructions, but a very complicated order which through its differently perpetual transformations and changes in their own meaningful ways evolves into some seemingly "catastrophic extinctions" only when there is a need for liquidation in its space-times of already outdated and thus dysfunctional kind of mass whether physical or spiritual (or anti-physical) forms, 
structures and individualities of the existence. Everything that exists (in terms of internal regenerating order of its transformations) already in contradiction with its just useful function becomes extinct because of certain processual causes and not from any inherent chaos within the processes of its basic Energy. And thanks to these extinctions other forms are free to reborn or take over prominence in advancing the original purpose and development of being. This is forever realized only by the anonymously operating substrate of the pure Energy (Te, De), present everywhere in the Multiverse/Universe/Cosmoses as well as in the reality of our Earth.

The modern global society, (if it is to survive on Earth for further millenniums) will need to reconcile with such reality and in accordance with it adopt quite a new worldview, neither materialistic nor monotheistic-religious or dogmatic, but one which will accept the truth that Humankind (as one of many living forms on Earth) must live here in a synergic harmony with the universal laws of Nature, and cannot overflow the banks of the limits of becoming a danger to the survival of our overall planetary entity! Because the Earth is in this solar system a self-regulated as well as self-cherishing living organism which anonymously expunge in its processes everything that begins to overreach the actual conditions required for its lasting, also Humankind is liable to adhere to this law and although each person here is functionally self-responsible part of the entire social organism of Humankind on Earth, all individuals (especially if one effects some useful processes of transformations here by his/her rationally educated judgment) - are given to cooperate with that living organism of Earth as the functional parts of global Humankind! A fortiori, when the human population on Earth begins too excessively multiply itself and overreach boundaries of exhaustions of the resources needed for our survival on Earth! For that reason this law, thanks to its basic antipodal (=yin-yang) contradictory working substrate of Energy, begins to initiate not only natural but also social disasters in every such a case of the overreaching its boundaries. Anonymous antipodal dialectical process of contradictory tensions generates (as it had every time in the history) also now, in our present reality, spawns entirely new, not only natural but also social inspirers of mass liquidation. Of course, it is a very cruel observation, but the law of liquidation of all what overflows own borders or limits is one of the basic laws of the natural processes of antipodal dialectical tensions in the Multiverse, and thus of course also here in our human society on Earth! In our reality it can be achieved not only by some terrible natural catastrophes but also by ideological, religious or political fanaticism which develops in individuals or groups as tools of their motivations what then brings into being the active actors in those liquidating processes on Earth.

Everyone now needs to rethink his/her conviction as well as the whole Humankind's behaviors on Earth and not place on the top of our priorities an abnormal material profit or unlimited reproduction of our population, but from the point of view of the earthly life's obligations put into the center of his/her interest the entire existence of Humankind, and thus the perfect maintenance of the whole complex of the proportional harmonization of natural, social and environmental conditions for our purposeful life on Earth. Instead of the "duty to God, Country, Company, or other artificial body" every one of us, the people, have our primary duty to fulfill all obligations for rescuing a sustainable life on Earth, including prevention against the potentially destructive effects of overpopulation.

True modern scientific research cannot be today already only analytical (and fragmented into the natural scientific and socio scientific fields), but synthesized from many common scientific points of view, progressively consequential, without being deformed by any religious, ideological or political demagogies. And thus not only Western but also some Far Eastern as well as ancient Chinese metaphysical and ethical conceptions, so different from ours, can inspire us to reach such standpoint.

We ought not to forget that the basic Energy of being is anonymous and does not know to grant any exceptions!!

\section{REFERENCES}

[1] Yi Jing, In Russian translation: SHUCKIJ, J. K: Kitajskaja klassicheskaja KNIGA PEREMEN (Classical Chinese Book of Changes), Vostochnaja literatura PAH Publishers, Moskva 1997.

[2] HEGEL, G. W.: Philosophie der Geschichte. In Slovak translation: VÁROSSOVÁ, E. (ed.): Novoveká racionalistická filozofia, Epocha Publishers, Bratislava 1970.

[3] ZEMPLINER, A.: Cínská filosofie v novověké evropské filosofii (Chinese Philosophy in Modern European Philosophy), Academia Publishers, Prague 1966.

[4] Encyclopaedia Wikipedia: Laws of Thermodynamics, https://en.wikipedia.org/wiki/Laws_of_thermodynamics.

[5] (Composite authors): Laozi jicheng (A Collection of Editions and Commentaries for the Laozi).15 volumes, Zongiiao wenhua chubanshi, Beijing 2011.

[6] Dao yuan“ in Huang-Lao si jing, ed. by Chang L. S. and Yu Feng (1998); or by Chen Guying (1995); as well as in Chen Yaoting's edition from Shanghai Academy of Social Sciences or The Taoist canon under the title: Zangwai daoshu, compiled by Hu Daojing. Bian zhu (a collective of compilers) in: Mawangdui han mubo shu, Wenwu chuban shi, Beijing 1980.

[7] http://www.kolej.mff.cuni.cz/ /motm275/skripta/mzahrad/no de158.html

[8] https://en.wikipedia.org/wiki/Uncertainty_principle

[9] LAOZI zong mu he Wang Pi zhu, in the book LAOZI, ZHUANGZI, No.9213 of Ssu-pu pei-yao, Chung-hua shu chü, Taipei 1965 (reprint).

[10] WENZI: Wenzi zuanyi. In No. 9221 of Ssu-pu pei-yao, Chung-hua shu chü, Taipei 1965 (reprint). 
[11] HAWKING, S. W.: A Brief History of Time. From the Big bang to Black Holes. (In Czech translation: Stručná historie času), Mladá Fronta Publishers, Prague 1991, ISBN 80-204-0169-5.

[12] (Composite authors): Laozi Jincheng (A Collection of Editions and Commentaries for the Laozi).15 volumes, Zongjiao wenhua chubanshi, Beijing 2011.

[13] HESHANG Kung: Dao De jing in Tao Te Ching, Zongjiao wenhua chuban shi, Beijing 2007.

[14] HESHANG Kung: Dao De Jing in ZHANG, Yunliang: Song Mashaben Laozi Dao De Jing, Gugong bo wu yuan, Shanghai 1932 (reprint 1991).

[15] BARROW, J.D.: The Book of nothing, Vintage Books of Random House, New York 2001, ISBN 0-375-72609-8.
[16] ČARNOGURSKÁ, M.: Lao c' a proces vzniku Tao Te tïngu (Laozi and the Process of the Genesis of Dao De jing), 2 Volumes, Veda Publishers, Bratislava 2009 and 2012, ISBN 978-80-224-1274-2.

[17] HEGEL, G. W.: Geschichte der Philosophie. In Czech translation: Dějiny filosofie Academia Publishers (Nakladatelství Československé Akademie Věd), Prague1961,

[18] GRIBBIN, J.: In Search of the Double Helix, Quantum physics and Life, (in Czech translation: Pátráni po dvojité šroubovnici), Columbus Publishers, Prague, 2006, ISBN 978-80-7249-193-3.

[19] GRIBBIN, J.: In Search of the Big Bang, (in Czech translation: Pátrání po Velkém třesku), Columbus Publishers, Prague 2002, ISBN 978-80-7249-046-X. 